\title{
CORRECTION
}

\section{Correction to: Differentiating societal costs of disability worsening in multiple sclerosis}

\author{
Nils-Henning Ness ${ }^{1} \cdot$ Dirk Schriefer $^{1} \cdot$ Rocco Haase $^{1} \cdot$ Benjamin Ettle $^{2} \cdot$ Christian Cornelissen $^{3} \cdot$ Tjalf Ziemssen $^{1}$ (i)
}

Published online: 16 January 2020

○) Springer-Verlag GmbH Germany, part of Springer Nature 2020

\section{Correction to: Journal of Neurology}

$$
\text { https://doi.org/10.1007/s00415-019-09676-4 }
$$

The original version of this article unfortunately contained a mistake. The captions of Figs. 2 and 3 are mismatched.

The correct captions of Figs. 2 and 3 are given in the following page.

The original article has been corrected.

The original article can be found online at https://doi.org/10.1007/ s00415-019-09676-4.

Tjalf Ziemssen

Tjalf.Ziemssen@uniklinikum-dresden.de

1 MS Center, Center of Clinical Neuroscience, Department of Neurology, University Hospital Carl Gustav Carus, Fetscherstr. 74, 01307 Dresden, Germany

2 Novartis Pharma GmbH, Nuremberg, Germany

3 Siemens Healthineers AG, Erlangen, Germany 
Fig. 2 Effects (IRR, 95\% CI) of PIRA and RAW on the quarterly economic costs compared to DAF. PIRA increased the quarterly total costs by $29 \%$ over 2 years compared to DAF. Results were adjusted for age, gender, EDSS, time and data source. PIRA progression independent of relapse activity, $R A W$ relapse-associated worsening, $D A F$ disease activity free. $* p<0.05 ; * * p<0.01$ (a) Total

RAW vs DAF**

(b) Direct medical

PIRA vs DAF*

RAW vs DAF**

(c) Direct non medical

PIRA vs DAF

RAW vs DAF*

(d) Indirect

PIRA vs DAF*

RAW vs DAF*
$1.29(1.08-1.53)$

$1.56(1.30-1.87)$

$1.48(1.13-1.95)$

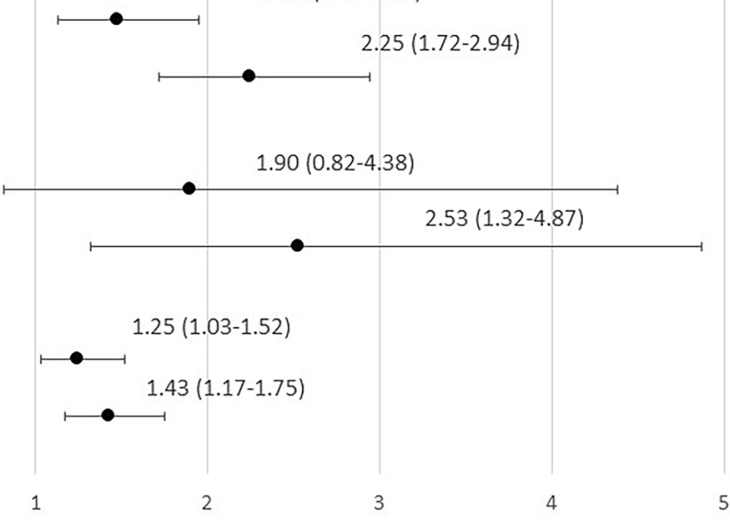

Incidence Rate Ratios (95\%-Confidence Intervals)

Fig. 3 Comparison of patients with in- and outpatient hospitalizations within 2 years. In the RAW population, the rate of patients with at least one inpatient hospital stay was almost twice as high compared to PIRA population (18\% vs. $32 \%)$

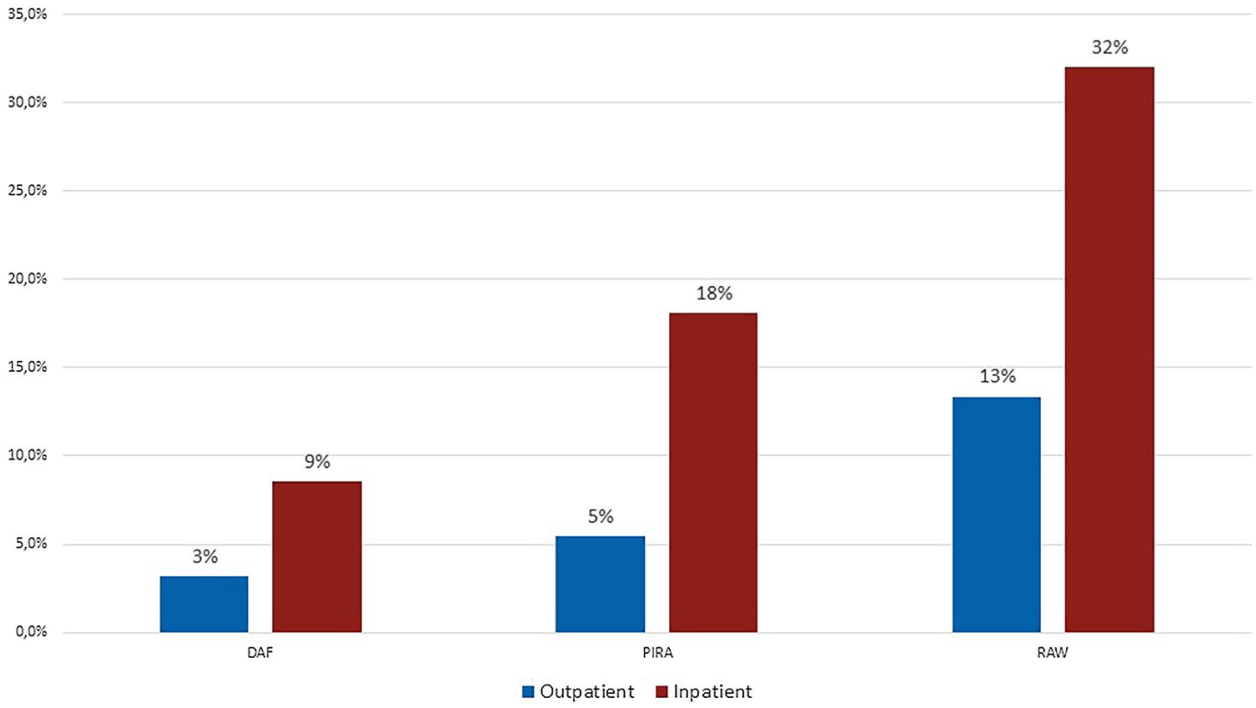

